\title{
Alternative Methods: 3Rs, Research and Regulatory Aspects
}

Francesca Caloni ${ }^{1}$, Patrizia Costa ${ }^{2}$, Gianni Dal Negro ${ }^{3}$, Isabella De Angelis ${ }^{4}$, Silvia Dotti ${ }^{5}$, Giovanna Lazzari ${ }^{6}$, Giacomo Metteucci ${ }^{7}$, and Augusto Vitale ${ }^{8}$

${ }^{1}$ Università degli Studi di Milano, Department of Health, Animal Science and Food Safety, Milan, Italy; ${ }^{2}$ Università degli studi di Milano, Laboratory Animal Science and Medicine Specialization School, Milan, Italy; ${ }^{3}$ GlaxoSmithKline R\&D Limited, UK; ${ }^{4}$ Istituto Superiore di Sanità, ISS, Department of Environment and Primary Prevention, Rome, Italy; ${ }^{5}$ Istituto Zooprofilattico Sperimentale della Lombardia e dell'Emilia Romagna "Bruno Ubertini” (IZSLER), Brescia, Italy; ${ }^{6}$ Avantea, Laboratory of Reproductive Technologies, Cremona, Italy; ${ }^{7}$ Università degli studi di Milano, LAS and Animal Welfare Consultant, Milan, Italy; ${ }^{8}$ Istituto Superiore di Sanità, ISS; Department of Cell Biology and Neuroscience, Section of Behavioural Neuroscience, Rome, Italy

A meeting on alternative methods to animal testing was held April 17-19 at the Brescia headquarters of the Istituto Zooprofilattico Sperimentale della Lombardia e Emilia Romagna. The subject is of crucial interest for the researchers' community because European Directive 63/2010/EU, which must be adopted by the Member States, draws specific attention to alternative methods. The workshop was split into sessions that dealt with different topics. On the first day, the presentations focused on the 3Rs Principle: Replacement, Reduction, and Refinement in animal research. The session "The principle of the $3 R$ in ethics and science: regulatory framework and dissemination," moderated by Dr Patrizia Costa, opened with the presentation of Dr Susanna Louhimies, a prominent figure in the European Commission, (DG Environment), who presented the state of transposition and implementation of Directive $63 / 2010 / \mathrm{EU}$ by and in the different Member States. Innovation, strengths, and goals emphasize the fact that the legal obligation of applying the principle of the $3 \mathrm{R}$ s results in a constructive ongoing debate among the different stakeholders at the European level and in a strong and consequent implementation and dissemination of the 3 Rs principles. Moreover, it was pointed out that Italy is one of the countries that have not yet implemented the regulation at the national level. Dr Giovanni Botta, Italian Ministry of Health, outlined the control and inspection activities carried out by the Ministry and the application of the principle of the 3Rs within those institutional activities. In his talk Dr Botta pointed out that the conditions of experimental animals in our country have improved over the last 10 years, and it is hoped that the new Directive will be signed into law as soon as possible. The activities of ECVAM and the new role of coordination in the implementation of alternative tests within the EU were presented by Dr Ingrid Langezaal. The presentation outlined the activities of the European center and described the alternative tests already validated and the process of making information exchange more efficient and dynamic. This cooperation will allow for the creation of a network between the centers of reference in the Member States, with the possibility of working in a coordinated and constructive manner. Dr Deborah Noviki of Novartis NVD Global Welfare Officer, explained the interest of the big pharma industry in the 3 Rs principle and how the principles are pragmatically applied.

On the second day, as part of the "in vivo methods" session moderated by Dr Giacomo Matteucci, Dr Augusto Vitale, ISS, highlighted the relationship between the principle of the 3Rs and good experimental practice. In particular, for each "R," a case study was illustrated in which the application of this concept led to improvement in experimental practice and in the results obtained. One of the models described is related to the study of asthma. This is a model derived from human tissue, and it is capable of explaining the relationship between the genetic background of the respiratory tract cells and the type of injury resulting from exposure to external agents. Moreover, it was pointed out that better cooperation and exchange of data, both nationally and internationally, would actually decrease the number of redundant experimental tests and, consequently, the total number of animals used. Finally, creating better living conditions for laboratory animals means obtaining more reliable and replicable results. Dr Guerino Lombardi, IZSLER, Brescia, explained the articles of Directive 2010/63 for the protection of animals used in scientific procedures, as related to the fairness and transparency of the experimental procedure. This correctness must respect both animal welfare and the scientific data collected. In this regard, the declaration in one of the introductory points of the Directive was mentioned. It asserts that animals are considered to be sentient beings and therefore consideration must be given to their capacity to experience pain and suffering. The presentation put particular emphasis on the importance of the cost/benefit analysis that must be implemented when considering a particular experimental protocol. Prof. Gabriele Bono from the Università di Padova made it clear that the concept of animal suffering has been present for some time and has influenced the evolution of animal ethics, which is the part of moral philosophy involving our behavior towards animals. Finally, the need for 
dialogue between those in favor of animal testing and those who do not consider it to be a justifiable practice was emphasized. Dr Christina von Hunolstein (ISS) underscored how the quality of a vaccine is regulated and monitored in all its phases, from registration to pharmaco-vigilance. Following Convention ETS 123, the European Pharmacopoeia Commission re-evaluated in vivo studies with the aim of applying the 3 Rs where possible. Safety and potency tests accepted by the Pharmacopoeia and "Official Medicine Control Laboratories" (OMCL) were described. The most important example is the test for neurovirulence of "Oral Poliovirus Vaccine" (OPV) where it was possible to apply both the concept of Refinement (from monkey to transgenic mouse) and Reduction, as OMCL observed the mice from the point of view of a manufacturer in an independent manner, without the need repeat the same test in its laboratories. Finally, entities that collaborate to eliminate in vivo testing of vaccines and keep up with the state of the art of alternative tests (EDQM, Group 15 of experts from Ph Eur, EMA, and WHO) were mentioned. Dr Lucia Luperi (RTC) highlighted parts of Directive 2010/63/EU that address both the 3 Rs and the complete replacement of tests on live animals for scientific and educational purposes. Furthermore, the principle of work implemented within a CRO that provides for the application of high quality standards and animal protection (BPL application and AAALAC accreditation) was illustrated. In particular, the procedures commonly used in the management and use of laboratory animals were pointed out, including the phases of health and behavioral management, both crucial for a correct approach to the use of animals. Finally, the role of the agencies in charge of animal welfare and of the International Conference on Harmonization (ICH, established in Paris in 1990) was presented. These consist of different organizations: American, European, and Japanese agencies and their respective inter-professional associations. These structures collaborate with the goal of harmonizing technical requirements for the registration of medicinal products for human use.

In the afternoon, the session "In vitro methods: new frontiers" moderated by Prof. Francesca Caloni, Università degli Studi di Milano, was focused on toxicology and the development of related in vitro methods with the talks of Prof. Enrico Sabbioni, ECSIN, Veneto Nanotech and Dr Paride Mantecca, Università di Milano Bicocca. In recent years, issues related to possible health and environmental risks from exposure to nanomaterials (NM) have been of great interest. In fact, it has been observed that materials normally considered inert for the animal organism may become toxic in the nanoparticle form, in which they can enter the organism and cause adverse reactions. In his presentation, Professor Sabbioni highlighted certain aspects of the role of the $3 \mathrm{Rs}$ in in vitro nanotoxicology research (Hartung and Sabbioni, 2011), cytotoxicity, and morphological transformation induced in mouse fibroblasts (Balb/3T3) by microparticles, nanoparticles, and cobalt ions. Dr Mantecca illustrated further in vitro models for the evaluation of specific mechanisms of NP action. The importance of the QSAR method, Quantitative Structure-Activity Relationship, and its application was the subject of the presentation by Dr Emilio Benfenati from Mario Negri Institute, through the description of a series of available computer programs such as: EPISuite (http://www.epa.gov/opptintr/exposure/pubs/episuite. htm), OECD Toolbox (http://www.oecd.org/env/ehs/risk-assessment/theoecdqsartoolbox.htm), Vega (http://www.vega-qsar.eu/) and the most recent ANTARES CE (http://www.antares-life.eu/). Endocrine Active Substances (EAS), biologically active substances that interact or interfere with the endocrine system causing a biological effect, were dealt with by Dr Stefano Lorenzetti, ISS. EAS are recognized to contain molecules that can cause adverse health effects and are therefore defined as endocrine disrupters (ED). Several European regulations (Rovida et al., 2013) suggest the use of alternative methods, without making use of animal tests, for identification of chemical substances placed in the market as EAS or ED, although adverse effects associated with endocrine disruption have not yet been defined. To this end, the development of in vitro methods may be aimed at the identification of action mechanisms associated with the molecular function such as tests of activation of gene transcription (ICATM, 2013) or cell-specific functionality tests such as that presented for the study of molecules with antiandrogen or androgen action in human prostate epithelial cells. Finally, Dr Alfonso Lostia, ECVAM, presented the SEURAT-1 project (Safety Evaluation Ultimately Replacing Animal Testing) funded by the European Commission through the call for submission "FP7-HEALTH-2010-Alternative-TestingStrategies" and by the European Cosmetics Association (Cosmetics Europe). This project consists of six complementary research sub-projects aimed at overcoming the lack of scientific knowledge needed to implement alternative safety tests that can flank and/or replace conventional animal testing due to repeated evidence of dose-based systemic toxicity. In addition, the initiative also is relevant in the context of the EU REACH Regulation (Registration, Evaluation, Authorization and Restriction of Chemicals), in the development of pharmaceuticals and other industrial sectors.

The third and final day revolved around the study of medical devices and their increasing use in medicine. Some of the most relevant aspects of the medical device (MD) were addressed in the session titled "In vitro methods and medical devices" moderated by Dr Isabella De Angelis, ISS. The common thread running through the presentations was the possibility of using alternative methods both in the assessment of conformity of the medical device as well as in the design phase of the latest generation of models. Dr Roberta Marcoaldi, ISS, presented in detail the regulatory approach to the marketing of medical devices. In her presentation, she pointed out that the European Community, with a view to promoting harmonization of legislation between Member States and given the broad category of products classified as MD, has limited the Essential Safety Requirements (ESR) that the MD placed in the Community market must meet to move freely within the Community (93/42/EEC and 2007/47/EC). The technical specifications of products meeting the ESR set out in the Directives are reported as harmonized standards, the application of which is voluntary; the manufacturer always can apply other technical specifications to meet the ESR. It is the task of the Notified Bodies to consider how the manufacturer has complied with the ESR and to authorize the release of the MD to the market. In this context, the use of alternative methods (in particular, methods of Replacement) is important as well and could be the subject of discussion between the manufacturer and the Notified Body. This possibility was described by Dr Marisa Meloni, Vit- 
roScreen, whose presentation focused on some in vitro tests suitable for assessing the toxicity and biocompatibility of MD. At the current level of knowledge, the regulated alternative methods are a particular and irreplaceable instrument for the identification of hazardous ingredients, but the validation of alternative methods for the evaluation of finished products which include MD, is not present in any EU Directive. Among the most promising in vitro methods for the assessment of MD safety, Dr Meloni highlighted: i) 3D in vitro human stratified epithelia (corneal epithelium, conjunctiva, oral mucosa, vaginal mucosa, nasal and bronchial epithelium, epidermis, intestinal epithelium); ii) percutaneous absorption and barrier integrity; iii) phototoxicity. In the presentation by Dr Silvia Dotti, IZSLER, the activities of the National Reference Center for Alternative Methods, Welfare and Care of Laboratory Animals, established by the Ministry of Health with Legislative Decree dated April 20, 2011 were described with particular focus on the subject of MD. The Center has extensive experience in: i) in vitro cytotoxicity tests; ii) tests for carcinogenicity; iii) preparation of reference standards.

Innovative aspects of tissue engineering, a growing sector of regenerative medicine and biomaterial science, were the subject of the second part of the session. Tommaso Sbrana, Engineeer, Piaggio center-Università di Pisa, introduced the guiding principles of tissue engineering and the use of bioreactors (Lanza et al., 2000). The reconstruction of in vitro tissue has as its main goal the formation of biological structures that can be used in vivo to replace those damaged and/or aged without having to resort to transplantation. To do this it is essential to simulate the physiological conditions that are the basis of tissue homeostasis as much as possible. For this purpose bioreactors, i.e., devices in which it is possible to perform biological processes under controlled conditions, can be used to mimic the physiological and pathological conditions of the tissue, reconstructing the three-dimensional structure and restoring the relationships between adjacent cells. In the presentation by Prof. Gerardo Catapano, Università della Calabria, the development of bioreactors for tissue engineering of bone was more specifically addressed. In this case, the studies were designed to optimize in vitro formation of bone substitutes, using human cells functionally similar to the in vivo counterpart. A further objective of the studies, but still at a preliminary stage, is the development of in vitro models for the study and treatment of bone diseases. Prof. Catapano subsequently outlined some of the key features necessary for ensuring that organs and tissue engineering are considered reliable from the biological point of view and can be used as alternative methods in the evaluation of the toxicity of drugs and chemicals.

The last session of the day, titled "In vitro methods for the evaluation of effectiveness of drugs," was moderated by Dr Gianni Dal Negro and Dr Maura Ferrari. In this session Dr Paolo Cavanni, APTUIT, gave an overview of the principal technologies used in drug research, both for the screening of new active ingredients and for the evaluation of their action mechanisms. These methods allow for the identification of new modulation schemes in terms of receptor activity. Dr Serena Cinelli, RTC, stressed the impor- tance of the development of in vitro tests during the pre-clinical phase of the study of new molecules. In vivo testing can thus be avoided when the data obtained prove to be accurate and can be standardized. Another subject discussed in this session was that of new cellular therapies that are currently under development. Dr Enrico Lucarelli, IOR, introduced mesenchymal stromal cells (MSCs) and their potential key role in regenerative medicine, although the speaker underlined that their capabilities/features need to be further clarified and developed. Cell therapy studies must be performed in vivo but there are also many aspects that can be studied in vitro first and then transferred to animals for further clinical investigation. It is expected that in the future new technologies will provide the required data using a smaller number of animals, and in vitro methods will be more extensively applied.

Finally, Prof. Francesco di Pisa, Università degli Studi di Siena, made a presentation focused on Reduction and the application of statistical methods for appropriate experimental design. This discipline is becoming increasingly important, not only to evaluate the performance of in vivo/in vitro tests but also ensures the proper use of animals. A correct scientific approach must involve the use of as few animals as possible: a goal that can be reached only by applying proper statistical methods in the experimental design.

In conclusion, this workshop highlighted the increasing need to implement and refine alternative methods. In fact, in all sessions, the speakers underscored the fact that technological and methodological advances are improving/increasing the possibilities of performing in vitro studies, therefore reducing the use of in vivo methods and ensuring a correct approach combining science and ethics.

\section{References}

Hartung, T. and Sabbioni, E. (2011). Alternative in vitro assays in nanomaterial toxicology. Wiley Interdiscip Rev Nanomed Nanobiotechnol 3, 545-573.

Lanza, R. P., Langer, R., and Vacanti, J. (eds.) (2000). Principles of Tissue Engineering ( $2^{\text {nd }}$ ed.). San Diego, CA, USA: Academic Press.

Rovida, C., De Angelis, I., and Lorenzetti, S. (2013). Alternative in vitro methods to characterize the role of Endocrine Active Substances (EASs) in hormone-targeted tissues. ALTEX 30, 253-255.

\section{Correspondence to}

Francesca Caloni, DVM PhD

Università degli Studi di Milano

Department of Health

Animal Science and Food Safety

Via Celoria 10,

20133 Milan - Italy

Phone: ++3902 50317884

Fax: ++3902 50317890

e-mail: francesca.caloni@unimi.it 